\title{
A Novel NMF Guided Level-set for DWI Prostate Segmentation
}

Patrick McClure $^{1 *}$, Fahmi Khalifa ${ }^{1,2 *}$, Ahmed Soliman ${ }^{1}$, Mohamed Abou El-Ghar ${ }^{3}$, Georgy Gimelfarb ${ }^{4}$, Adel Elmagraby $^{6}$ and Ayman El-Baz ${ }^{1 *}$

${ }^{1}$ Biolmaging Laboratory, Department of Bioengineering, University of Louisville, Louisville, KY 40292, USA

${ }^{2}$ Electronics and Communication Engineering, Faculty of Engineering, University of Mansoura, Mansoura 35516, Egypt

${ }^{3}$ Radiology Department, Urology and Nephrology Center, University of Mansoura, Mansoura 35516, Egypt

${ }^{4}$ Department of Computer Science, University of Auckland, Auckland 1142, New Zealand

${ }^{5}$ Computer Engineering and Computer Science Department, University of Louisville, Louisville, KY 40292, USA

${ }^{6}$ Information Systems Department, King Saud University, KSA

\begin{abstract}
Objective: To develop an automated 3D framework for prostate segmentation from diffusion-weighted imaging (DWI).

Methods: The proposed framework integrates level-set deformable model and nonnegative matrix factorization (NMF) techniques. In the proposed framework, the level-set is guided by a novel speed function that is derived using NMF, which extracts meaningful features from a large dimensional feature space. The NMF attributes are calculated using information from the DWI intensity, a probabilistic shape model, and the spatial interactions between prostate voxels. The shape model is constructed using a set of training prostate volumes and is then updated during the segmentation process using an appearance-based method that takes into account both a voxel's location and its intensity value. The spatial interactions are modeled using a second-order pairwise 3D Markov-Gibbs random field (MGRF).

Results: We tested our framework on 125 in vivo DWI-MRI prostate data sets that has been collected from 10 subjects at b-values ranging from 0 to $1000 \mathrm{~s} / \mathrm{mm}^{2}$. Our results show that using NMF-based feature fusion to guide the level-set increases the segmentation accuracy compared with previously proposed methods using two evaluation metrics, the dice similarity coefficient (DSC) and Hausdorff distance (HD). The proposed method achieved an average DSC of $0.868 \pm 0.03$ and an average HD of $5.61 \pm 2.12 \mathrm{~mm}$ compared to an average DSC of $0.83 \pm 0.07$ and an average $\mathrm{HD}$ of $6.67 \pm 1.84 \mathrm{~mm}$ for a maximum a posteriori (MAP)-based level-set method, and an average DSC of $0.810 \pm 0.05$ and an average HD of $9.07 \pm 1.64$ for a level-set driven only by intensity and shape information.

Conclusions: Experimental results show that the integration of 3D intensity, shape, and spatial features with NMF-based feature fusion increases the ability of the proposed method to perform robust prostate segmentation despite image noise, inter-patient anatomical differences, and the similar intensities of the prostate and surrounding tissues.
\end{abstract}

Keywords: DWI; level-set; Prostate; Segmentation; NMF; MGRF

\section{Introduction}

Prostate localization is an essential step for developing a medical image-based framework for prostate analysis (e.g., a computer aided diagnostic (CAD) system for diagnosing prostate cancer). As such, several techniques have been proposed for segmenting the prostate from a variety of magnetic resonance imaging (MRI) modalities. However, accurate prostate segmentation for MRI data can be challenging due to image noise, inter-patient anatomical differences, and the similar intensities of the prostate and surrounding tissues (e.g., the bladder). To overcome these challenges, different segmentation techniques have been proposed. Deformable models (DMs) have been particularly popular, especially active shape models (ASMs) [1] and level-sets [2]. For example, Allen et al. [3] proposed a DM framework for three dimensional (3D) prostate segmentation from T2-MRI. The DM evolution was controlled by voxel intensity and a statistical shape model. A hybrid 2D/3D ASM-based methodology for 3D MRI prostate segmentation was proposed by Zhu et al. [4]. Additionally, Ghose et al. [5] used a similar approach that aligned T2-MRI data then used an active appearance model (AAM), an extension of ASM, guided by appearance and shape information to segment the prostate. Also, Gao et al. [6] aligned MR images before segmenting the prostate using a level-set guided by appearance information and a learned shape prior. In addition, Martin et al. [7] used a probabilistic anatomical atlas to constrain a DM-based framework for segmenting the prostate from $3 \mathrm{D}$ T2-MR images.
Statistical-based techniques have also been utilized for segmenting prostates from MRI data. For example, Ghose et al. [8] proposed a probabilistic graph-cut-based framework for 3D T2-MRI prostate segmentation based on a probabilistic atlas. Firjany et al. [9] proposed a Markov random field (MRF) image model [10] for 2D dynamic contrast enhanced (DCE)-MRI prostate segmentation that combined a graphcut approach with a prior shape model of the prostate and the visual appearance of the prostate image, modeled using a linear combination of discrete Gaussians (LCDG) [11]. Their method was later extended in $[12,13]$ to allow for $3 \mathrm{D}$ prostate segmentation from DCE-MRI data. A maximum a posteriori (MAP) [14]-based framework that performed

*Corresponding authors: Ayman El-Baz, Lutz Hall, Room 423, University of Louisville, Louisville, KY 40292, USA, Tel: 502-852-5092; Fax: 502-852-6806; E-mail: aselba01@louisville.edu

Patrick McClure, Room 423, Biolmaging Laboratory, Department of Bioengineering University of Louisville, Louisville, KY 40292, USA

Fahmi Khalifa, Electronics and Communication Engineering, Faculty of Engineering, University of Mansoura, Mansoura 35516, Egypt

Received July 26, 2014; Accepted August 20, 2014; Published August 22, 2014

Citation: McClure P, Khalifa F, Soliman A, El-Ghar MA, Gimelfarb G, et al. (2014) A Novel NMF Guided Level-set for DWI Prostate Segmentation. J Comput Sci Syst Biol 7: 209-216. doi:10.4172/jcsb.1000158

Copyright: $\odot 2014 \mathrm{McCl}$ ure $\mathrm{P}$, et al. This is an open-access article distributed under the terms of the Creative Commons Attribution License, which permits unrestricted use, distribution, and reproduction in any medium, provided the original author and source are credited. 
automated 3D MRI prostate segmentation using an MRF model and statistical shape information was proposed by Makni et al. [15]. Also, Klein et al. [16] presented an atlas-based segmentation approach to extract the prostate from MR images based on averaging the best atlases that match the image to be segmented. Another technique, proposed by Dowling et al. [17] used an automated atlas approach to segment the prostate region based on a selective and iterative method for performance level estimation (SIMPLE) [18] based alignment technique.

In addition to DMs and statistical-based techniques, several other methods have been proposed to segment the prostate from MR images. Flores-Tapia et al. [19] proposed a semi-automated edge detection technique for MRI prostate segmentation based on a static wavelet transform [20] to locate the prostate edges. Also, a semi-automated approach by Vikal et al. [21] used a prostate shape prior to detect the contour in each slice and then refined them to form a 3D prostate surface. Additionally, Random walk classification [22] was used for MRI prostate segmentation by Khurd et al. [23].

In addition to T2-MRI and DCE MRI, diffusion weighted imaging (DWI) MRI has emerged as a promising image modality for prostate analysis in recent years due to its ability to measure both structural and functional qualities. Additionally, acquiring DWI does not require injecting a patient with a contrast agent, unlike DCE-MRI. Specifically for prostate cancer diagnosis, the apparent diffusion coefficient (ADC), a feature calculated using DWI images acquired at different magnetic field strengths (or b-values), has been found capable of discriminating between malignant and benign prostate tissues [24-30]. As a result of the discriminative nature of $\mathrm{ADC}$ values, DWI prostate localization is a required step in many prostate cancer CAD systems. A limited number of DWI prostate segmentation techniques have been proposed to overcome the challenges listed above. For example, Firjani et al. proposed the use of a MAP [13] method using intensity, shape and spatial features for 3D DWI prostate segmentation [24,31,32]. The intensity was modeled using the LCDG model and a 3D Markov-Gibbs random field (MGRF) was used as the spatial model. Also, Liu et al. [33] proposed a 2D level-set guided by intensity and shape information for DWI prostate segmentation. The intensity of a pixel was found by taking the average of the pixel's intensity at a b-value of $0 \mathrm{~s} / \mathrm{mm}^{2}$ and the ADC value found using b-values of 0 and $100 \mathrm{~s} / \mathrm{mm}^{2}$. The shape model of the prostate was determined by fitting an ellipse to an initial segmentation generated using Otsu intensity thresholding [34]. Similarly, Liu et al. developed a 3D level-set method guided by intensity and shape information [35]. The intensity of a voxel was the ADC value found using $b$-values of 0 and $600 \mathrm{~s} / \mathrm{mm}^{2}$. An initial segmentation was performed using a 3D level-set guided by this intensity information. The shape of the prostate was then approximated by fitting an ellipsoid and a series of ellipses to the initial segmentation. This intensity and shape information was then used to guide another 3D level-set for final segmentation. While these methods have successfully performed DWI prostate segmentation, they can be sensitive to similarities between the intensities of object and background voxels.

To overcome the above challenges, a novel deformable model-based framework for prostate segmentation from DWI data is proposed. Our framework utilizes a novel nonnegative matrix factorization (NMF) feature fusion method to create a more robust model for guiding the evolution of a 3D level-set deformable model for DWI prostate segmentation. The proposed NMF-based feature fusion approach incorporates three features, namely DWI intensity, prostate shape, and spatial interactions between prostate voxels. The features generated by this technique are then used to guide the evolution of a 3D level-set deformable model to extract the prostate from DWI data (Figure 1).

\section{Methods}

In this paper, a novel level-set-based framework for DWI prostate segmentation Figure 1 is proposed. Geometric deformable models based on level-sets are commonly used techniques for object segmentation from medical imaging. Level-sets have been successfully applied to segment several organs in the human body (e.g., kidney $[36,37]$ heart $[38,39]$ and prostate $[6,33,35]$. In the definition of the level-set, the evolving surface at any time instant $t$ is represented by the zero level, $\phi_{n}(x, y, z)=0$, of an implicit function, namely a distance map of the signed minimum Euclidean distance from each voxel to the surface.

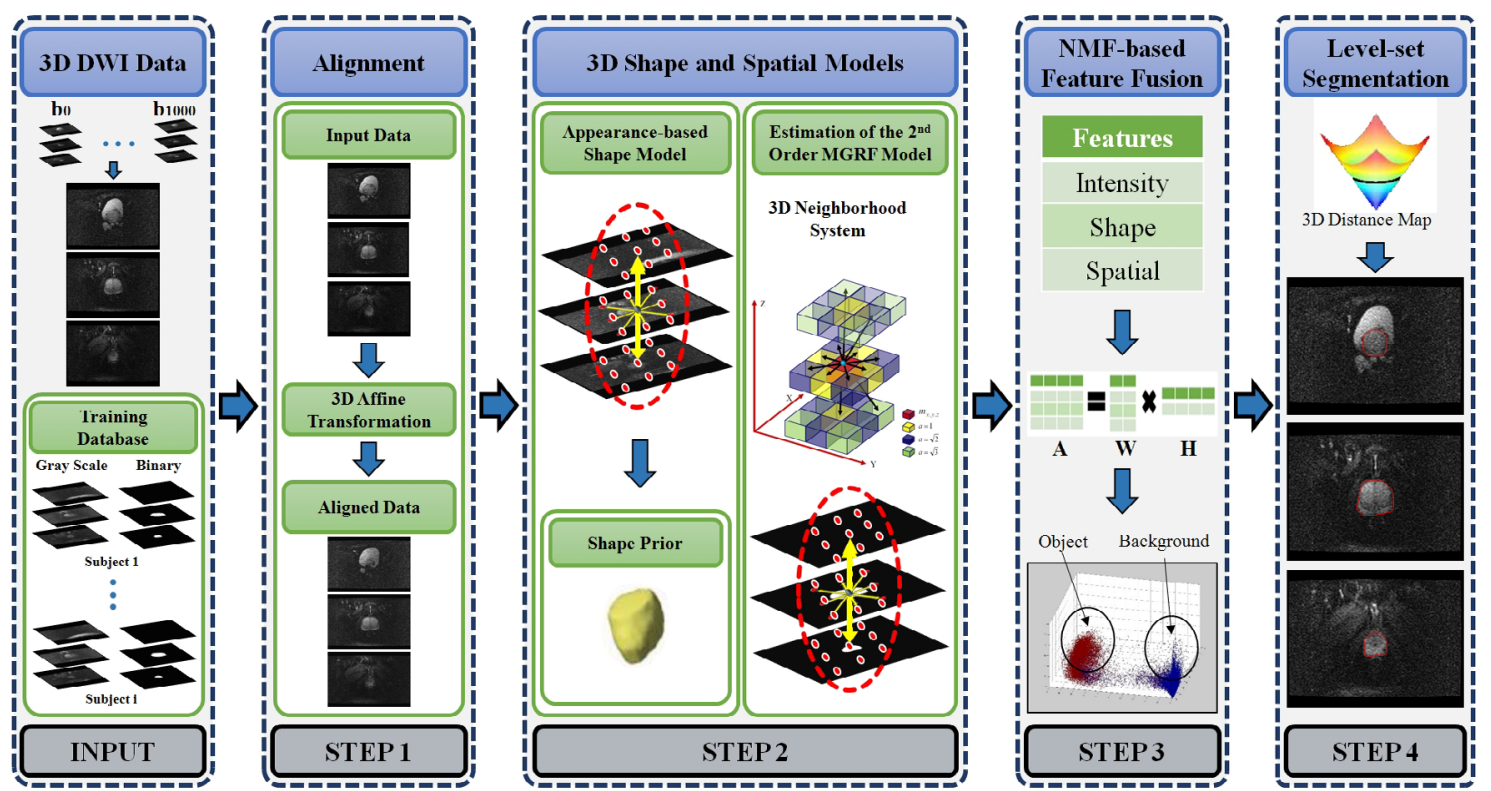

Figure 1: A diagram of the proposed framework for the 3D segmentation of the prostate on diffusion-weighted imaging (DWI) data. 
This formulation results in points inside the surface having negative (or positive) values and voxels outside the surface having positive (or negative) values, respectively. Mathematically, the evolution of the level-set is defined by [2]

$$
\phi_{n+1}(x, y, z)=\phi_{n}(x, y, z)-\tau \boldsymbol{V}_{n}(x, y, z)\left|\nabla \phi_{n}(x, y, z)\right|
$$

where $t$ is the discrete time instant $t=n \tau$ taken with a step $\tau, \tau>0$ and $\nabla=\left[\frac{\partial}{\partial x}, \frac{\partial}{\partial y}, \frac{\partial}{\partial z}\right]$ is the differential operator. This evolution is guided by the speed function $V_{n}(x, y, z)[40]$.

Previous speed functions that use image intensities, object edges, and gradient vector flow have had difficulty segmenting noisy images and those with poor object-background contrast. More effective speed functions have been developed by using shape models to incorporate prior information of the object of interest. However, this has not completely overcome image in homogeneities (e.g., large image noise and discontinuous object boundaries). Recent techniques utilize stochastic speeds that incorporate object-background shape and appearance models [36,41-45]. In order to more accurately segment the prostate from DWI data, we propose a novel speed function that depends on regional statistics derived from the prostate and background regions. Namely, it takes into account regional appearance, shape, and spatial features of the DWI data. These features are combined using an NMF-based fusion method to provide the voxel-wise guidance of the deformable model. Details of these features and their NMF fusion are given in the following sections. First, a summary of the basic notations used throughout this paper is provided.

\section{Basic notation}

Let $\mathbf{Q}=\{0, \ldots, Q-1\}$ and $\mathbf{L}=\{0,1\}$ be the set of $Q$ integer gray levels and a set of object (1) and background (0) labels, respectively. Also, let a $3 \mathrm{D}$ arithmetic lattice $\mathbf{R}=\{(x, y, z): 0 \leq x \leq X-1 ; 0 \leq y \leq Y-1 ; 0 \leq z \leq$ $Z-1\}$ support the grayscale DWI data $\mathbf{g}: \mathbf{R} \rightarrow \mathbf{Q}$ and their binary region maps $\mathbf{m}: \mathbf{R} \rightarrow \mathbf{L}$. Each voxel $(x, y, z)$ is associated with its neighbors,

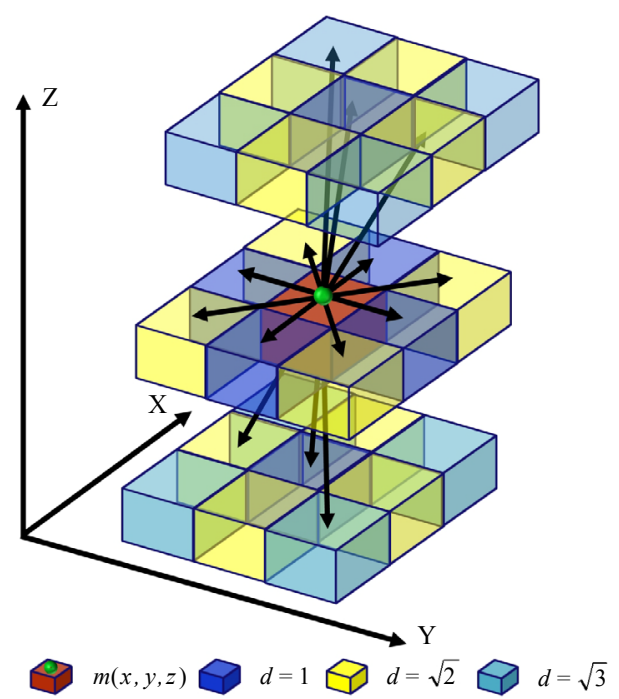

Figure 2: Illustration of a voxels 26 neighborhood system for the 3D intensity and the 3DMarkov-Gibbs random filed (MGRF) spatial model. Here, d represents the Euclidean distance between the central voxel $m_{x, y, z}$ and its neighbors. $\{(x+\xi, y+\eta, z+\zeta):(x+\xi, y+\eta, z+\zeta) \in \mathbf{R} ;(\xi, \eta, \zeta) \in \mathbf{N}\}$ where $\mathbf{N}$ is the 26 neighborhood system Figure 2 defined by $\xi \in\{-1,0,1\}, \eta \in\{-1,0,1\}$ and $\zeta \in\{-1,0,1\}$.

\section{D Appearance, shape, and spatial features}

Appearance-based shape model: Most prostates have a similar near-ellipsoidal shape [46]. As a result, the inclusion of a shape prior can significantly improve the segmentation accuracy. In the pro-posed framework, an appearance-based shape model is built that takes into account both a voxel's location and its intensity information. A shape database was constructed by co-aligning training data sets using a 3D affine transformation with 12 degrees of freedom ( 3 for 3D translation, 3 for $3 \mathrm{D}$ rotation, 3 for $3 \mathrm{D}$ scaling, and 3 for $3 \mathrm{D}$ shearing) and maximizing mutual information (MI) [47]. A shape prior is a spatially variant independent random field of region labels for the co-aligned data. Mathematically, this is defined as:

$$
\boldsymbol{P}_{s h}(\boldsymbol{m})=\prod_{(\boldsymbol{x}, \boldsymbol{y}, z) \in \boldsymbol{R}} \boldsymbol{P}_{s h: x, y, z}\left(m_{\boldsymbol{x}, \boldsymbol{y}, z}\right)
$$

where $P_{\text {sh:x:y;z }}(l)$ is the voxel-wise empirical probability for label $l \in$ L. For each input DWI volume to be segmented, the shape prior is constructed by a process guided by the visual appearance features of the DWI data. The appearance-based shape prior is then estimated using the method summarized in Algorithm 1.

Algorithm 1: Algorithm for Calculating an Appearance-based Shape Model

Calculate the value of the shape prior probability at each voxel using the following steps:

1. Transform each test subject voxel to the shape database domain using the calculated $3 \mathrm{D}$ affine transformation matrix $(\mathrm{T})$.

2. Initialize an $N_{1 i} \times N_{2 i} \times N_{3 i}$ search space centered at the voxel.

3. Find voxels inside the search space with corresponding gray levels to the center voxel in all training data sets.

4. If no corresponding voxels are found, increase the search space size and repeat the previous step.

5. Calculate the label probabilities for each voxel based on the relative occurrence of each label in the search results.

Spatial voxel interaction model: In addition to the prostate shape prior, analyzing the interactions of a voxel and its neighbors can improve segmentation $[24,44]$. In order to model these interactions, a second-order 3D MGRF model [48] is used. The MGRF model of the region map $\mathbf{m}$ is defined as:

$$
\boldsymbol{P}_{s p}(\boldsymbol{m})=\frac{1}{\boldsymbol{Z}_{N}} \exp \sum_{(\boldsymbol{x}, \boldsymbol{y}, \boldsymbol{z}) \in \boldsymbol{R}(\xi, \eta, \zeta) \in N} \sum_{\boldsymbol{e q}}\left(m_{\boldsymbol{x}, \boldsymbol{y}, z}, m_{\boldsymbol{x}+\boldsymbol{\xi}, \boldsymbol{y}+\boldsymbol{\eta}, z+\zeta}\right)
$$

where $V_{e q}\left(m_{x ; y ; z}, m_{x+\xi ; y+\eta ; z+\zeta}\right)$ is the Gibbs potential and $Z_{N}$ is the normalization factor which can be approximated as [49]:

$$
\boldsymbol{Z}_{N} \approx \exp \sum_{(\boldsymbol{x}, \boldsymbol{y}, \boldsymbol{z}) \in \boldsymbol{R}(\xi, \eta, \zeta) \in N} \sum_{\boldsymbol{e q}}\left(m_{\boldsymbol{x}, \boldsymbol{y}, \boldsymbol{z}}, m_{\boldsymbol{x}+\boldsymbol{\xi}, \boldsymbol{y}+\boldsymbol{\eta}, \boldsymbol{z}+\zeta}\right)
$$

The MGRF used can be viewed as a 3D extension of the autobinomial, or Potts, model with the exception that the Gibbs potential is estimated analytically. The maximum likelihood estimate of the potential is given as [50]: 


$$
V_{e q}=2\left(f_{e q}(\boldsymbol{m})-\frac{1}{2}\right)
$$

Where $f_{e q}(\mathbf{m})$ is the relative frequency of equal labels in the voxel pairs $((x, y, z),(x+\xi, y+\eta, z+\zeta))$.

\section{NMF-based feature fusion}

NMF is a method for extracting meaningful features from data sets for representing different classes in the data [50]. This is done by calculating a weight matrix $W$ that transforms a vector from the input space into a new feature space ( $H$-space) through factorizing the input matrix $A$. NMF has been applied to various data analysis problems such as document clustering [51] and facial recognition [52]. In addition, it has been used in a few segmentation systems. Particularly, Xie et al. [53] used NMF to segment the spinal cord, corpus callosum, and hippocampus regions of rats from diffusion tensor imaging (DTI) by $\mathrm{k}$-means clustering the column vectors of the produced $H$ matrix. Also, Sandler et al. [53] proposed using NMF to factorize intensity histogram data for generic image segmentation.

In this paper, an NMF-based approach is proposed to find the weights for each feature in order to create a feature space where object and background classes are better separated, dimensionality is reduced, and information from the training data set is encoded. NMF factorizes a $k$ by $n$ input matrix $A$ into a $k$ by $r$ weight matrix $W$, which contains the basis vectors of the new space as columns, and an $r$ by $n$ output matrix $H$ where $k$ is the dimensionality of the input column vectors, $n$ is the number of input and output column vectors, and $r$ is the dimensionality of the output column vectors [50]. Mathematically, this is defined as:

$$
\boldsymbol{A} \approx \boldsymbol{W H}
$$

where $W$ and $H$ are calculated by minimizing the Euclidean distance between $A$ and $W H$ with the constraint that $W$ and $H$ contain only nonnegative values. This results in the constrained optimization problem:

$$
\begin{aligned}
& \min _{\boldsymbol{W}, \boldsymbol{H}} \frac{1}{2} \boldsymbol{A}-\boldsymbol{W H} \\
& \text { subject to } \boldsymbol{W}, \boldsymbol{H}>0
\end{aligned}
$$

In the literature, several methods have been used to optimize this function. The most prominent methods have been multiplicative gradient descent, alternating least square (ALS), and projected gradient descent (PGD) [55]. In this paper, the multiplicative method [55] is used because of its ease of implementation. This method iteratively updates $W$ and $H$ until convergence using the following rules:

$$
\begin{gathered}
\boldsymbol{H}_{\alpha \beta} \leftarrow \boldsymbol{H}_{\alpha \beta} \frac{\left(\boldsymbol{W}^{\boldsymbol{T}} \boldsymbol{A}\right)_{\alpha \beta}}{\left(\boldsymbol{W}^{\boldsymbol{T}} \boldsymbol{W H}\right)_{\alpha \beta}} \\
\boldsymbol{W}_{\gamma \alpha} \leftarrow \boldsymbol{W}_{\gamma \alpha} \frac{\left(\boldsymbol{A} \boldsymbol{H}^{\boldsymbol{T}}\right)_{\gamma \alpha}}{\left(\boldsymbol{W H} \boldsymbol{H}^{\boldsymbol{T}}\right)_{\gamma \alpha}}
\end{gathered}
$$

where $\alpha: 1 \rightarrow r, \beta: 1 \rightarrow n$, and $\gamma: 1 \rightarrow k$.

In the proposed framework, NMF is performed on a matrix that has a $k^{\text {th }}$ dimensional, one dimension for each calculated feature, column vector for each voxel $(x, y, z)$ in the training volumes. The input features are the intensity values of the voxel $(x, y z)$ and its neighbors, the spatial interactions between voxel $(x, y, z)$ and its neighbors, and the value of the shape prior at $(x, y, z)$. The $W$ resulting from factorization is used as the basis vectors to transform new feature vectors into the new $r$-dimensional space ( $H$-space). Also, the resulting $H$ is used to find the $r$-dimensional centroids corresponding to the object and background classes using k-means clustering [57]. For each voxel in a testing volume, a $k^{\text {th }}$ dimensional feature vector was calculated. This resulted in a $k$ by $n$ feature matrix $B$ where $n$ is the number of voxels in the volume. The new $r$ dimensional vectors corresponding to the input voxels are calculated by multiplying $B$ by the psuedo-inverse of $W$, which can be replaced by $W^{T}$ assuming orthogonality of the columns of $W$ [58]. Mathematically, this is described as:

$$
\boldsymbol{H}_{B}=\boldsymbol{W}^{T} B
$$

\section{Regional speed function}

In this paper, a novel speed function to control the evolution of the level-set deformable model is proposed. This speed function is derived using the NMF-based fusion of DWI features, $H_{B: x ; y z z}$ for voxel $(x, y, z)$. The proposed speed function $V_{n}(x, y, z)$ is defined as $V_{n}(x, y, z)=\kappa \vartheta(x$, $y, z)$, where $\kappa$ is the curvature and $\vartheta(x, y, z)$ is defined as:

$$
\vartheta(x, y, z)=\left\{\begin{array}{c}
-E_{o b j: x ; y ; z} \text { if } E_{o b j: x ; y ; z}>E_{b g: x ; y ; z} \\
E_{b g: x ; y ; z} \text { otherwise }
\end{array}\right.
$$

Here, $E_{\text {obj:x;y;z }}=P_{n m f x ; y ; z}(1)+P_{\text {sh: } x ; y ; z}(1)+P_{\text {sp: } x ; y ; z}(1)$ where $P_{\text {sh: } x ; y ; z}(1)$ is the object shape prior probability and $P_{\text {sp:xyyzz.z. }}(1)$ is the object MGRF model probability. Similarly, $E_{b p: x ; y ; z}=P_{n m f: x ; y ; z z}(0)+P_{\text {sh: } x ; y ; z}(0)+P_{\text {sp: } x ; y ; z}(0)$ where $P_{\text {sh: } x ; y ; z}(0)$ is the background shape prior probability and $P_{\mathrm{sp}: x ; y ; z ;}(0)$ is the background MGRF model probability. $P_{n m f x x y ; z}(1)$ and $P_{n m f x ; y ; z}(0)$ are defined using the distances from the two class centroids to the $H$-space vector of voxel $(x, y, z)$. The overall segmentation framework is summarized by Algorithm 2.

$$
D S C=\frac{2 T P}{2 T P+F P+F N}
$$

The value of the DSC ranges from 0 to 1 , where 0 means that there is no similarity and 1 means that there is perfect similarity.

Algorithm 2: Proposed Algorithm for DWI Prostate Segmentation

Segment the prostate from a DWI volume by:

1. Align the input DWI volume with the training database using the MI-based affine transformation.

2. Calculate the appearance-based shape prior using Algorithm 1.

3. Calculate the $3 \mathrm{D}$ pairwise voxel interactions Equation 3.

4. Perform NMF-based feature fusion Equations 6 and 7.

5. Calculate the probabilities that each voxel is object or background using the NMF-based features.

6. Use these probabilities to guide the evolution of a level-set to segment the prostate Equation 11.

\section{Performance Evaluation Metrics}

The performance of the proposed segmentation framework was evaluated using two accuracy metrics: (1) Dice similarity coefficient (DSC) and (2) Hausdorff distance (HD). Generally, there are two ways to evaluate any segmentation approach. The first determines the similarity between model segmentation and the gold standard, i.e., ground truth. The most commonly used metric is the dice similarity coefficient (DSC). The DSC is useful for determining the false positive 
Citation: McClure P, Khalifa F, Soliman A, El-Ghar MA, Gimelfarb G, et al. (2014) A Novel NMF Guided Level-set for DWI Prostate Segmentation. J Comput Sci Syst Biol 7: 209-216. doi:10.4172/jcsb.1000158

and false negative segmentation, which are important for volumetric analysis. The second way determines the distance between the borders of a model segmentation and the ground truth. We used the Hausdorff distance to measure the closeness of our segmentation to the ground truth. This particular type of metric is important for shape analysis application. Therefore, we used both types metric to evaluate the accuracy of our proposed segmentation approach. These metrics are detailed below.

\section{Dice similarity coefficient (DSC)}

Many segmentation and classification metrics are based on the determination of true positive (TP), false positive (FP), true negative (TN), and false negative (FN) values, Figure 3 . The TP is the number of correctly positively labeled samples; the FP is the number of incorrectly positively labeled samples; the TN is the number of correctly negatively labeled samples; and the FN is the number of incorrectly negatively labeled samples. These values can be used to calculate the DSC as shown by [59]:

\section{Hausdorff distance (HD)}

Distance measures are another type of performance metric used for evaluating segmentation methods. The Euclidean distance is often utilized, but another common measure is the HD. The HD from a set $\mathbf{A}_{1}$ to a set $\mathbf{A}_{2}$ is defined as the maximum distance of the set $\mathbf{A}_{1}$ to the nearest point in the set $\mathbf{A}_{2}$ [60] (Figure 4):

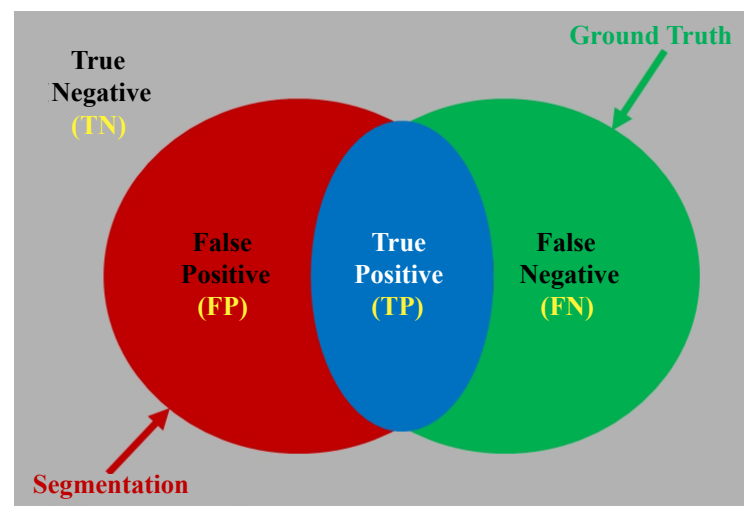

Figure 3: Schematic illustration of the measuring the segmentation errors for calculating the Dice similarity coefficient (DSC).

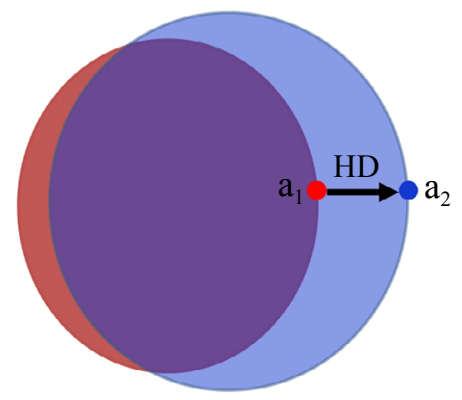

$$
\text { Boundary } A_{1} \square \text { Boundary } A_{2}
$$

Figure 4: Schematic illustration of measuring the 2D Hausdorff distance (HD) between two boundaries, $A_{1}$ and $A_{2}$.

$$
\boldsymbol{H D}\left(A_{1}, A_{2}\right)=\max _{a_{1} \in A_{1}}\left\{\min _{a_{2} \in A_{2}}\left\{d\left(a_{1}, a_{2}\right)\right\}\right\}
$$

where $a_{1}$ and $a_{2}$ are points of sets $\mathbf{A}_{1}$ and $\mathbf{A}_{2}$, respectively, and $d$ $\left(a_{1}, a_{2}\right)$ is Euclidean distance between these points. The bidirectional Hausdorff distance, denoted by $H D_{B i}(\mathbf{G T}, \mathbf{S R})$, between the segmented region (SR) and its ground truth $(\mathbf{G T})$ is defined as:

$$
H D_{B i}(G T, S R)=\max \left\{H D(G T, S R), H D_{B i}(S R, G T)\right\}
$$

The smaller the distance, the better the segmentation method performed. The ideal case with perfect segmentation is when the bidirectional Hausdorff distance is equal to 0 .

\section{Experimental Results}

\section{Medical images}

Our frameworks have been tested on 125 data sets that have been collected from 10 subjects at different b-values. These data sets were acquired using a scanner (SIGNA Horizon, General Electric Medical Systems, Milwaukee, WI) with the following parameters: TE: $84: 6 \mathrm{~ms}$; TR: $8.000 \mathrm{~ms}$; FOV $32 \mathrm{~cm}$; slice thickness $3 \mathrm{~mm}$; inter-slice gap $0 \mathrm{~mm}$; and two excitations. The DWI volumes were collected with b-values ranging from 0 to $1000 \mathrm{~s} / \mathrm{mm}^{2}$ using a voxel size of $1.25 \times 1.25 \times 3.00$ $\mathrm{mm}^{3}$. The ground truth segmentations used in training and in verifying the segmentation results were manually created by an MR expert.

\section{Segmentation results}

The proposed NMF-based approach has been tested on the abovementioned data sets. Sample cross sections of the 3D segmentations of 4 subjects are shown in (Figure 5) for volumes acquired at $b_{200}$. The accuracy of the proposed prostate segmentation approach is evaluated using both the DSC [58] and the HD [60] metrics to determine the
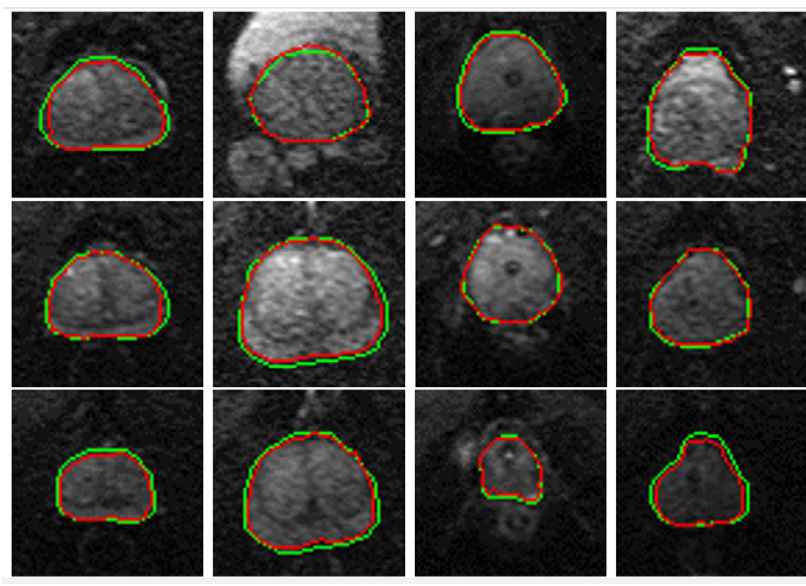

Figure 5: Sample segmentation results presented in 2D for visualization of the 3D segmentation performed by the proposed nonnegative matrix factorization (NMF)-based level-set approach at different cross sections for 5 different subjects. The green and red colors correspond respectively to the ground truth and our segmentation.

\begin{tabular}{|c|c|c|}
\hline Method & DSC $($ Mean \pm SD) & HD (Mean \pm SD) \\
\hline Our Approach & $0.868 \pm 0.03$ & $5.61 \pm 2.12 \mathrm{~mm}$ \\
\hline MAP [25] & $0.834 \pm 0.07$ & $6.67 \pm 1.84 \mathrm{~mm}$ \\
\hline Liu et al. [35] & $0.810 \pm 0.05$ & $9.07 \pm 1.64 \mathrm{~mm}$ \\
\hline
\end{tabular}

Table 1: Segmentation accuracies for the proposed nonnegative matrix factorization (NMF)-based approach and the compared approaches using the Dice similarity coefficient (DSC) and the Hausdorff distance (HD) metrics. Note that "SD" stands for standard deviation. 

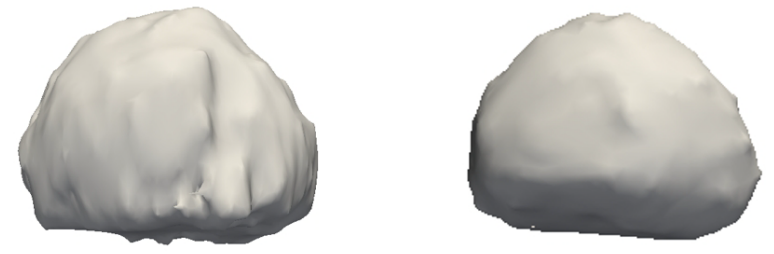

Figure 6: 3D visualizations of the segmented prostate object generated using our approach for two subjects.

$\mathrm{NMF}$
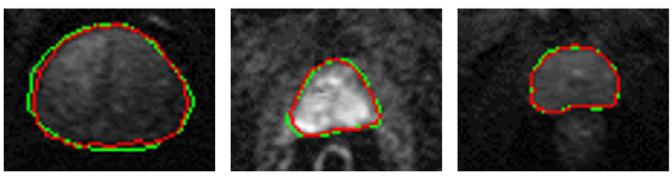

MAP
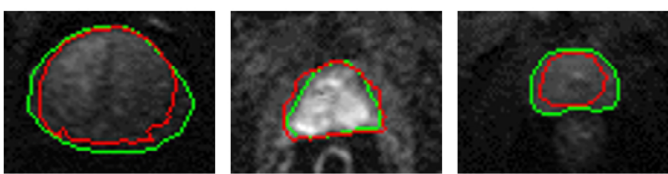

Figure 7: Comparative segmentation results projected into 2D for visualization of the 3D segmentation for 3 different patients using the (a) proposed approach and (b) the maximum a posteriori (MAP) guided level-set where the green and red curves correspond to the ground truth and the segmentations, respectively.
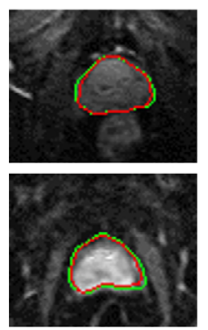

$b_{100}$
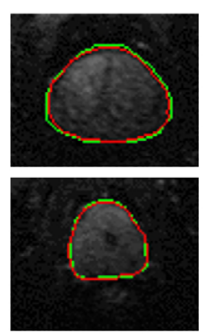

$b_{300}$
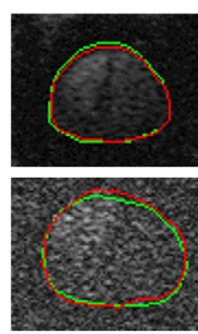

$b_{600}$
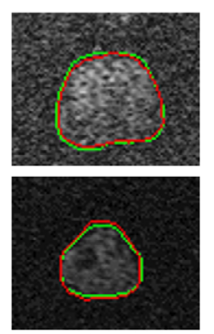

$b_{800}$
Figure 8: Sample receiver operating characteristic (ROC) curves for the proposed (red) and the maximum a posteriori (MAP)-based (blue) level-set segmentation approaches.

agreement between our segmentation and the ground truth, as described in Section III. Table 1 summarizes the DSC and HD statistics obtained for all test data sets. The ground truth was obtained by manual contouring of the prostate borders by an MR expert. The final $3 \mathrm{D}$ segmentations obtained by the proposed approach for two of the prostates in the data set are shown in Figure 6.

To highlight the advantage of integrating the NMF-based fusion of the three image features (i.e., the 3D appearance, spatial, and shape features) into the level set segmentation. The results using our approach were compared to: $(i)$ the reported results for the 3D approach developed by Liu et al. [35] and (ii) the results of a level-set guided by the MAP model proposed by Firjani et al. [24] that utilized the probability that a voxel was object or background based on its intensity, shape, and spatial information. The MAP-based approach by Firjani et al. [24] demonstrated higher accuracy compared with the approach proposed in [35]. Figure 7 qualitatively compares the accuracy of the NMF-based and the MAP-based level-set methods for sample independent subjects at different cross sections, with respect to the ground truth.
Moreover, the average DSC and HD values of all three of the compared methods are shown in Table 1. As demonstrated in Table 1 the use of the proposed NMF-based level-set method has improved the accuracy of the segmentation, as evidenced by the higher DSC and smaller $\mathrm{HD}$ values. These results highlight the advantages of integrating the NMF-based fusion of the 3D appearance, shape, and spatial interaction features to guide the evolution of the level set function.

In addition to $\mathrm{DSC}$ and $\mathrm{HD}$, another common metric for evaluating segmentations is the receiver operating characteristic (ROC) [61,62]. The ROC measures the sensitivity of a segmentation using different classification thresholds by demonstrating the interaction between the ration of the TP and FP rates. The area under the ROC curve ranges from 0 to 1 , with 1 corresponding to an ideal segmentation. Sample ROC curves of the NMF and MAP guiding forces, as well as the areas under the curves $(\mathrm{Az})$, are shown in Figure 8.

In recent years, diffusion-weighted MR has shown an increased use in CAD systems for prostate cancer diagnosis by estimating diffusion parameters (e.g., apparent diffusion coefficient) using DWI data acquired at both higher and lower b-values [24-30]. However, segmenting DWI data acquired at higher b-values is a challenge compared with those at lower b-values due to the decreased contrast between the object and the background. In contrast to the existing approaches, the proposed framework possesses the ability to accurately extract the prostate from DWI data at higher b-values. This is due to the integration of the $3 \mathrm{D}$ appearance, shape, and spatial features with an NMF feature fusion that increases the robustness of the proposed approach to overcome large image noise at higher b-values. The segmentation of DWI data at higher b-values for 8 subjects is shown Figure 9. As demonstrated in this figure, the robustness proposed approach produce precise segmentation of the prostate at higher b-values compared with the ground truth.

\section{Conclusion}

In this paper, a novel framework for the $3 \mathrm{D}$ segmentation of the prostate from diffusion-weighted MR images is proposed. The proposed framework utilizes a nonnegative matrix factorization (NMF) approach to combine different image features, namely the 3D appearance, shape, and spatial features to guide a level-set deformable model to extract the prostate from DWI. Experimental results on in-vivo data sets at different b-values showed a significantly better segmentation of the prostate using the proposed NMF-based feature fusion than using either MAP with the same input information or intensity and shape information alone. This in part can be explained by the fact that the addition of NMF-based feature fusion allows the proposed method to perform robust prostate segmentation despite image noise, inter-patient anatomical differences, and the similar intensities of the prostate and surrounding tissues. In future work, we plan to utilize the proposed segmentation technique in a complete computer-aided diagnostic (CAD) system for prostate cancer detection using DWI-MRI. Therefore, this segmentation framework will be tested with a larger data set in order to verify its robustness.

\section{References}

1. Cootes TF, Taylor CJ (1992) Active shape models: smart snakes. Proceedings of the British Machine Vision Conference 266-275.

2. Osher S, Sethian JA (1988) Fronts propagating with curvature-dependent speed: algorithms based on Hamilton-Jacobi formulations. Journal of Computational Physics 79: 12-49.

3. Allen PD, Graham J, Williamson DC, Hutchinson CE (2006) Differential segmentation of the prostate in MR images using combined $3 D$ shape 
modelling and voxel classification. Proceedings of the 3rd IEEE International Symposium on Biomedical Imaging 410-413.

4. Zhu Y, Williams S, Zwiggelaar R (2007) A hybrid ASM approach for sparse volumetric data segmentation. Pattern Recognition and Image Analysis 17: 252-258

5. Ghose S, Oliver A, Marti R, Llado X, Freixenet J, et al. (2012) A hybrid framework of multiple active appearance models and global registration for 3D prostate segmentation in MRI. Proceedings of the SPIE Conference on Medical Imaging.

6. Gao Y, Sandhu R, Fichtinger G, Tannenbaum AR (2010) A coupled global registration and segmentation framework with application to magnetic resonance prostate imagery. IEEE Trans Med Imaging 29: 1781-1794.

7. Martin S, Troccaz J, Daanenc V (2010) Automated segmentation of the prostate in 3D MR images using a probabilistic atlas and a spatially constrained deformable model. Med Phys 37: 1579-1590.

8. Ghose S, Mitra J, Oliver A, Marti R, Llado X, et al. (2012) Graph cut energy minimization in a probabilistic learning framework for 3D prostate segmentation in MRI. Proceedings of the $21^{\text {st }}$ International Conference on Pattern Recognition

9. Firjany A, Elnakib A, El-Baz A, Gimel'farb G, El-Ghar M, et al. (2010) Novel Stochastic Framework for Accurate Segmentation of Prostate in Dynamic Contrast Enhanced MRI. Proceedings of the First International Workshop on Prostate Cancer Imaging: Computer-Aided Diagnosis, Prognosis, and Intervention 121-122.

10. Li SZ, Singh S (2009) Markov random field modeling in image analysis.

11. El-Baz A (2006) Novel Stochastic Models for Medical Image Analysis University of Louisville.

12. Firjani A, Elnakib A, Khalifa F, Gimmel'farb G, Abo El-Ghar M, et al. (2011) A new 3D automatic segmentation framework for accurate segmentation of prostate from DCE-MRI. Proceedings of the IEEE International Conference on Image Processing 1476-1479.

13. Firjani A, Elnakib A, Khalifa F, Gimel'farb G, Abo El-Ghar M, et al. (2011) A new 3D automatic segmentation framework for accurate segmentation of prostate from DCE-MRI. Proceedings of the IEEE International Symposium on Biomedical Imaging (ISBI'11): 1476-1479.

14. Mark BL, Turin W (2011) Probability, Random Processes, and Statistical Analysis. Cambridge University Press Textbooks

15. Makni N, Puech P, Lopes R, Dewalle AS, Colot O, et al. (2009) Combining a deformable model and a probabilistic framework for an automatic 3D segmentation of prostate on MRI. Int J Comput Assist Radiol Surg 4: 181-188.

16. Klein S, van der Heide UA, Lips IM, van Vulpen M, Staring M, et al. (2008) Automatic segmentation of the prostate in $3 \mathrm{D}$ MR images by atlas matching using localized mutual information. Med Phys 35: 1407-1417.

17. Dowling JA, Fripp J, Chandra S, Pluim JPW, Lambert J, et al. (2011) Fas automatic multi-atlas segmentation of the prostate from 3D MR images. Prostate Cancer Imaging 10-21.

18. Langerak TR, van der Heide UA, Kotte AN, Viergever MA, van Vulpen M, et al (2010) Label fusion in atlas-based segmentation using a selective and iterative method for performance level estimation (SIMPLE). IEEE Trans Med Imaging 29: $2000-2008$

19. Flores-Tapia D, Thomas G, Venugopal N, McCurdy B, Pistorius S (2008) Semi automatic MRI prostate segmentation based on wavelet multiscale products. Proceedings of the 30th Annual International Conference of the IEEE Engineering in Medicine and Biology Society 3020 - 3023.

20. Pesquet JC, Krim H, Carfantan H (1996) Time-invariant orthonormal wavelet representations. IEEE Transactions on Signal Processing 44: 1964-1970.

21. Vikal S, Haker S, Tempany C, Fichtinger G (2009) Prostate contouring in MRI guided biopsy. Proc SPIE 7259: 72594A.

22. Grady L (2006) Random walks for image segmentation. IEEE Trans Pattern Anal Mach Intell 28: 1768-1783.

23. Khurd P, Grady L, Gajera K, Diallo M, Gall P, et al. (2011) Facilitating 3D spectroscopic imaging through automatic prostate localization in MR images using random walker segmentation initialized via boosted classifiers. Prostate Cancer Imaging 47-56.
24. Firjani A, Elnakib A, Khalifa F, Gimel'farb G, El-Ghar MA, et al. (2013) A diffusion-weighted imaging based diagnostic system for early detection of prostate cancer. Journal of Biomededical Science and Engineering 6: 346-356.

25. Litjens G, Vos P, Barentsz J, Karssemeijer N, Huisman H (2011) Automatic computer aided detection of abnormalities in multi-parametric prostate MRI. SPIE Medical Imaging.

26. Vos PC, Barentsz JO, Karssemeijer N, Huisman HJ (2012) Automatic computer-aided detection of prostate cancer based on multiparametric magnetic resonance image analysis. Phys Med Biol 57: 1527-1542.

27. Ozer S, Haider MA, Langer DL, van der Kwast TH, Evans AJ, et al. (2009) Prostate cancer localization with multispectral MRI based on relevance vector machines. Proceedings of the IEEE International Symposium on Biomedical Imaging: From Nano to Macro 73-76.

28. Niaf E, Rouvière O, Mège-Lechevallier F, Bratan F, Lartizien C (2012) Computer-aided diagnosis of prostate cancer in the peripheral zone using multiparametric MRI. Phys Med Biol 57: 3833-3851.

29. Peng Y, Jiang Y, Yang C, Brown JB, Antic T, et al. (2013) Quantitative analysis of multiparametric prostate MR images: differentiation between prostate cance and normal tissue and correlation with Gleason score--a computer-aided diagnosis development study. Radiology 267: 787-796.

30. Hambrock T, Vos PC, Hulsbergen-van de Kaa CA, Barentsz JO, Huisman HJ (2013) Prostate cancer: computer-aided diagnosis with multiparametric 3-T MR imaging--effect on observer performance. Radiology 266: 521-530.

31. Firjani A, Khalifa F, Elnakib A, Gimmel'farb G, Abo El-Ghar M, et al. (2011) 3D Automatic Approach for Precise Segmentation of the Prostate from DiffusionWeighted Magnetic Resonance Imaging. Proceedings of the IEEE International Conference on Image Processing 2285-2288.

32. Firjani A, Elnakib A, Khalifa F, Gimel'farb G, Abo El-Ghar M, et al. (2011) A new 3D automatic segmentation framework for accurate extraction of prostate from diffusion imaging. Proceedings of the Biomedical Sciences and Engineering Conference 1-4.

33. Liu X, Langer D, Haider M, Van der Kwast T, Evans A, et al. (2009) Unsupervised segmentation of the prostate using MR images based on level set with a shape prior. Conf Proc IEEE Eng Med Biol Soc 3613-3616.

34. Otsu N (1975) A threshold selection method from gray-level histograms Automatica 11: 23-27.

35. Liu X, Haider MA, Yetik IS (2011) Unsupervised 3D prostate segmentation based on diffusion-weighted imaging MRI using active contour models with a shape prior. Journal of Electrical and Computer Engineering 906-915

36. Khalifa F, Beache GM, El-Ghar MA, El-Diasty T, Gimel'farb G, et al. (2013) Dynamic contrast-enhanced MRI-based early detection of acute renal transplant rejection. IEEE Trans Med Imaging 32: 1910-1927.

37. Khalifa F, Gimel'farb G, El-Ghar MA, Sokhadze G, Manning S, et al. (2011) A new deformable model-based segmentation approach for accurate extraction of the kidney from abdominal CT images. Proceedings of the IEEE International Conference on Image Processing 3393-3396.

38. Khalifa F, Beache GM, Gimel'farb G, Giridharan GA, El-Baz A (2012) Accurate automatic analysis of cardiac cine images. IEEE Trans Biomed Eng 59: 445455

39. Khalifa F, Beache GM, Gimel'farb G, El-Baz A (2011) A novel approach for accurate estimation of left ventricle global indexes from short-axis cine MRI. Proceedings of IEEE International Conference on Image Processing 26452648

40. Osher S, Fedkiw R (2006) Level set methods and dynamic implicit surfaces New York: Springer-Verlag.

41. Khalifa F, Elnakib A, Beache G, Gimel'farb G, El-Ghar M, et al. (2011) 3D Kidney Segmentation from CT Images Using a Level Set Approach Guided by a Novel Stochastic Speed Function. Fichtinger G, Martel A, Peters T, editors. Proceedings of the International Conference on Medical Image Computing and Computer-Assisted Intervention 14: 587-94

42. Khalifa F, Beache GM, Elnakib A, Sliman H, Gimelfarb G, et al (2013) A new shape-based framework for the left ventricle wall segmentation from cardiac first-pass perfusion MRI. Proceedings of the International Symposium on Biomedical Imaging 41-44

43. Khalifa F, Beache GM, Nitzken M, Gimel'farb G, Giridharan GA, et al. (2011) 
Citation: McClure P, Khalifa F, Soliman A, El-Ghar MA, Gimelfarb G, et al. (2014) A Novel NMF Guided Level-set for DWI Prostate Segmentation. J Comput Sci Syst Biol 7: 209-216. doi:10.4172/jcsb.1000158

Automatic analysis of left ventricle wall thickness using short-axis cine CMR images. Proceedings of IEEE International Symposium on Biomedical Imaging From Nano to Macro 1306-1309.

44. Khalifa F, El-Baz A, Gimelfarb G, Ouseph R, El-Ghar MA (2010) Shapeappearance guided level-set deformable model for image segmentation. Proceedings of IAPR International Conference on Pattern Recognition 45814584.

45. Khalifa F, Beache G, El-Baz A, Gimelfarb G (2010) Deformable model guided by stochastic speed with application in cine images segmentation. Proceedings of IEEE International Conference on Image Processing 1725-1728.

46. Shier D, Butler J, Lewis R (2006) Hole's essentials of human anatomy and physiology. McGraw-Hill New York.

47. Viola PA, Wells III WM (1997) Alignment by Maximization of Mutual Information International Journal on Computer Vision 24: 137-154.

48. El-Baz A, Soliman A, McClure P, Gimelfarb G, El-Ghar MA, et al. (2012) Early assessment of malignant lung nodules based on the spatial analysis of detected lung nodules. Proceedings of the IEEE International Symposium on Biomedical Imaging: From Nana to Macro (ISBI'12).

49. Farag AA, El-Baz AS, Gimelfarb G (2006) Precise segmentation of multimodal images. IEEE Trans Image Process 15: 952-968.

50. Lee DD, Seung HS (1999) Learning the parts of objects by non-negative matrix factorization. Nature 401: 788-791.

51. Shahnaz F, Berry MW, Pauca VP, Plemmons RJ (2006) Document clustering using nonnegative matrix factorization. Information Processing \& Management 42: 373-386.

52. Zafeiriou S, Tefas A, Buciu I, Pitas I (2006) Exploiting discriminant information in nonnegative matrix factorization with application to frontal face verification IEEE Trans Neural Netw 17: 683-695

53. Xie Y, Ho J, Vemuri BC (2011) Nonnegative factorization of diffusion tensor images and its applications. Inf Process Med Imaging 22: 550-561.

54. Sandler R, Lindenbaum M (2011) Nonnegative Matrix Factorization with Earth Mover's Distance Metric for Image Analysis. IEEE Trans Pattern Anal Mach Intell.

55. Berry MW, Browne M, Langville AN, Pauca VP, Plemmons RJ (2007) Algorithms and applications for approximate nonnegative matrix factorization Computational Statistics and Data Analysis. 52:155-173.

56. Lee DD, Seung HS (2000) Algorithms for non-negative matrix factorization. Advances in neural information processing systems.

57. Duda RO, Hart PE, Stork DG (2012) Pattern classification. John Wiley \& Sons.

58. Hyvarinen A (1999) Sparse code shrinkage: denoising of nongaussian data by maximum likelihood estimation Neural Comput 11: 1739-1768.

59. Zou KH, Warfield SK, Bharatha A, Tempany CM, Kaus MR, et al. (2004 Statistical validation of image segmentation quality based on a spatial overlap index. Acad Radiol 11: 178-189.

60. Babalola KO, Patenaude B, Aljabar P, Schnabel J, Kennedy D, et al. (2009) An evaluation of four automatic methods of segmenting the subcortical structures in the brain. Neuroimage 47: 1435-1447.

61. Gerig G, Jomier M, Chakos M Valmet (2001) A new validation tool for assessing and improving 3D object segmentation. Medical Image Computing and Computer Assisted Intervention 516-523.

62. Hanley JA, McNeil BJ (1982) The meaning and use of the area under a receiver operating characteristic (ROC) curve. Radiology 143: 29-36. 